\title{
DEVELOPMENT OF SYSTEM FOR STABILIZING MOVEMENT OF POWER UNIT WITH REAR MOUNTED MOWER
}

\author{
Lilia Savchenko, Oksana Achkevych, Vasyl Achkevych, Tetyana Slipukha \\ National University of Life and Environmental Sciences of Ukraine, Ukraine \\ lilya_savchenko@ukr.net, achkevych@gmail.com, achkevychv@gmail.com,dubrova17@ukr.net
}

\begin{abstract}
The article is devoted to solving the problems of increasing the stability of motion of a power unit with a rear mounted mower. An analytical review of methods and means of ensuring the stability of the header movement of the power unit is given. Variational principles of mechanics are applied in the study of the dynamics of motion of a power unit. The regularity of the oscillatory angular displacement of the unit relative to the power unit has been established. An automated system is proposed for ensuring the stability of the movement of a power unit with a rear mounted mower. This system can be developed on the basis of the synthesis of mechanical and hydraulic subsystems with the activation of the drive to the field wheel. Equation describing the transmission ratio provides efficient automatic torque control on the field wheel of the implement. A decrease in the coefficient reduces the response of the system to external disturbances from resistance forces and unit deflection, while an increase can lead to an undesirable oscillatory mode of operation. It was found that the trajectories of movement of both serial and experimental units are characterized by the presence of constant components of deviations. According to Student's criteria, the introduction of an active drive to the field wheel reduces the trend value from 7.36 to 5.31. It has been substantiated that the active drive on the field wheel of the unit stabilizes the movement of the power unit. The root mean square deviation of the trajectory of movement decreases from $4.9 \mathrm{~cm}$ to $4.4 \mathrm{~cm}$, and the angle of deviation of the trajectory from the straight-line decreases on average by $10^{\circ}$. According to the results of testing the experimental power unit using an active drive on the field wheel of the unit, it increases the productivity by $6 \%$ with a decrease in the fuel consumption of about $9 \%$.
\end{abstract}

Keywords: rear mower, stability, automated system, drive.

\section{Introduction}

Agricultural production technologies during field work in a number of cases provide for the side asymmetric hitch of the agricultural machine relative to the energy tool. With this configuration of the machine-tractor unit (MTU), additional reactive forces are arising from the moments, creating a number of difficulties in the management of the unit.

The problem of the stability of the movement of mobile technical systems is devoted to a large number of studies [1-3]. In [4], the author leads an analysis of the instability of the movement of the wide-capture tractor and definite deviations from the trajectory. This leads to damage to plants and significantly depends on the scale of transverse deviations of the working equipment. The article offers methods for determining the kinematic parameters of wide-span tractors and the probability of damage to plants in a row. The choice of the distance between the kinematic center of the tractor and the center of resistance of the unit used for laying the controlled traffic is substantiated. In work [5] experimental researches of parameters of the plow unit are made according to the "push-pull" scheme on the basis of the HTZ-16131 tractor, from a position of satisfactory stability of its movement. In [6], an analysis of the stability of the tractor with a plow is also given, when an additional moment of decomposition acts on the plow, under the action of which the tractor can move away from the straightness of the movement. The paper presents a mathematical model of the tractor deflection due to external eccentric load on the hook, which allows to calculate the maximum value of the shear force.

In the work [7-9] is considered the straightness of the plow depending on the change of the design parameters. The objective of this study was to measure the draft, vertical, and lateral forces acting on the moldboard plow, a para-plow without a wing, para-plow with forward-bent wing, and a para-plow with a backward-bent wing at three working depths and three forward speeds in clay loam soil to investigate the use of a suitable para-plow instead of the moldboard plow.

In the dynamics of MTU movement, it is balanced on the verge of equilibrium, any even minor deviation from this position leads to its lateral displacements. As a rule, the equilibrium position without the use of special technical measures is achieved through the skill of the aggregate management. Thus, MTU layout solutions with a side hitch cannot be considered perfect both in terms of energy costs and in terms of the process efficiency. The existing experience of operation of such mobile units with asymmetric load distribution shows that there are various technical means of compensation efforts. They 
are usually associated with the differentiation of the distribution of the torque and propulsion with the complication of the drive. In this regard, it is important to ensure the stability and controllability of the MTU movement.

\section{Materials and methods}

Variation methods of analytical mechanics were used to study the kinematic and force characteristics of a dynamic system, numerical methods for solving the system of differential equations of motion of a machine-tractor unit, as well as methods of mathematical modeling and statistical analysis, trajectory characteristics.

A serial harvester was used for experimental research. In addition, changes are made to its design related to the development of an automatic control system (Fig. 1) and a power drive on the field support wheel (Fig. 2).

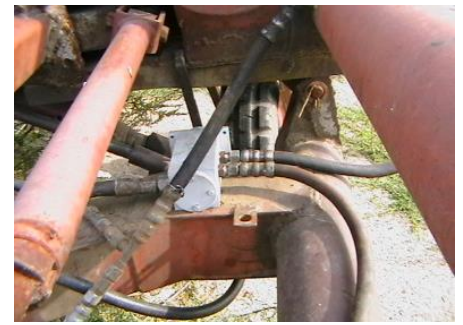

Fig. 1. Connection of the hydraulic distributor to the control system of the hydraulic motor on the field support wheel

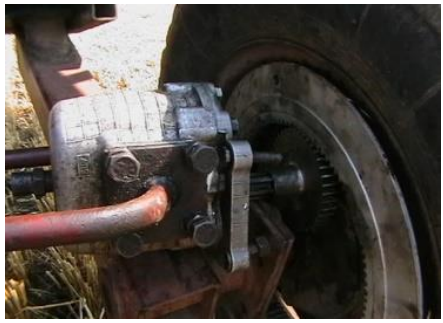

Fig. 2. General view of the hydraulic drive on the field support wheel of the harvester

The kinematic scheme of the MTU harvester, as a technical system integrated into a single whole, as well as the applied forces, moments and geometric dimensions are presented in Fig.3.

The study of the dynamics of the MTU harvesting movement is possible on the basis of the use of variational principles of mechanics. The general form of the equation for the harvesting MTU, taking into account the holonomy of the bonds, is presented as (1):

$$
\frac{d}{d t}\left(\frac{d T}{d \dot{q}_{s}}\right)-\frac{d T}{d q_{s}}=Q_{s}
$$

where $T$ - kinetic energy of the system;

$q_{s}-$ generalized coordinates;

$S$ - generalized coordinate index;

$Q_{s}$ - generalized force (moment) corresponding to the accepted generalized coordinate.

The kinetic energy of the MTU harvester consists of the kinetic energy of the carrier (tractor) and the kinetic energy of the aggregate agricultural machine (harvester). In turn, the kinetic energy of each of the components of the technical system is formed by gradual and rotational movement. Therefore, in general, the kinetic energy of AIT can be written as the following sum (2):

$$
\left.T=\sum_{i=1}^{2} T_{i}=0,5 \sum_{\mathrm{i}=1}^{2}\left[m_{i}\left(\dot{\chi}_{\mathrm{i}}+\dot{y}_{\mathrm{i}}^{2}\right)+I_{\mathrm{i}} \omega_{\mathrm{i}}^{2}\right)\right]
$$

Since we consider a two-mass system consisting of a tractor and a harvester, based on the general definition of kinetic energy, equation (1) can be written (3).

$$
T=\frac{1}{2} m_{t}\left(\dot{\chi}^{2}{ }_{t}+\dot{y}_{t}{ }_{t}\right)+\frac{1}{2} I_{t} \omega_{t}{ }^{2}+\frac{1}{2} m_{m}\left(\dot{\chi}^{2}{ }_{m}+\dot{y}_{m}{ }_{m}\right)+\frac{1}{2} I_{m} \omega_{m}{ }^{2},
$$

where $m_{t}-$ tractor weight;

$x_{t}, y_{t}-$ coordinates of the center mass of the tractor;

$I_{t}$ - moment of inertia of the tractor;

$\omega_{t}-$ angular velocity of rotation of the tractor around its own center of mass;

$m_{m}$ - harvester mass;

$x_{m}, y_{m}$ - coordinates of the center of gravity of the harvester;

$I_{m}$ - moment of inertia of the harvester;

$\omega_{m}-$ angular speed rotation of the harvester around its own center mass. 
The components $\dot{x}_{t}, \dot{x_{m}}, \dot{y_{t}}, \dot{y_{m}}$ of velocities are based on the geometric parameters of the construction of the MTU. The coordinates $x_{c t}, y_{c t}$ are taken as a basis of reference. According to them, the coordinates of the center of mass of the harvester are determined by the equations (4-7):

$$
\left\{\begin{array}{c}
x_{c m}=x_{c t}-\left(l_{1}-a_{1}\right) \cdot \sin \beta_{c t}-a_{2} \cdot \sin \beta_{c m}-b_{2} \cdot \cos \beta_{c m} \\
y_{c m}=y_{c t}-\left(l_{1}-a_{1}\right) \cdot \cos \beta_{c t}-a_{2} \cdot \cos \beta_{c m}+b_{2} \cdot \sin \beta_{c m}
\end{array}\right.
$$

where $a_{1}$ - distance between the front axle of the tractor and the center of mass of the tractor.

After the transformations, the equation takes the form:

$$
\begin{aligned}
& \left\{\begin{array}{l}
\ddot{x}_{c t}\left(m_{t}+m_{m}\right)+b_{2} m_{m} \ddot{\beta}_{c m}=\sum F_{x} \\
\ddot{y}_{c t}\left(m_{t}+m_{m}\right)+m_{m}\left[\left(l_{1}-a_{1}\right) \ddot{\beta}_{c t}+a_{2} \ddot{\beta}_{c m}\right]=\sum F_{\mathrm{y}}
\end{array}\right. \\
& I_{t} \cdot \ddot{\beta}_{c t}+m_{m}\left(l_{1}-a_{1}\right)\left(\ddot{x}_{c m} \sin \beta_{c t}-\ddot{y}_{c m} \cos \beta_{c t}\right)= \\
& M_{c t}-M_{o n}+\left(l_{1}-a_{1}\right)\left[F_{x 02} \sin \beta_{c t}-F_{\mathrm{y} 02} \cos \beta_{c t}\right] \\
& \ddot{\beta}_{\mathrm{cm}}\left(I_{m}+a_{2} m_{m} b_{2}+a_{2}^{2} m_{m}\right)+a_{2} m_{m}\left[\ddot{x}_{c t}+\ddot{y}_{c t}+\left(l_{1}-\right.\right. \\
& \left.\left.-a_{1}\right) \ddot{\beta}_{c t}\right]+\dot{\beta}_{c m}^{2} m_{m} a_{2}\left(1+\beta_{c m} b_{2}\right)+m_{m} \dot{\beta}_{c m}\left[b_{2}\left(\dot{x}_{c t}-\left(l_{1}-a_{1}\right) \dot{\beta}_{c t}+a_{2} \dot{y}_{c t}\right]=\sum M\right.
\end{aligned}
$$

Thus, the general dynamics of the MTU as a complex technical system is described by a total of four second-order differential equations (4), (5), (6). The first two of these equations (5) describe the translational motion of the system in the direction of the received coordinate axes $X$ and $Y$. The third equation (6) establishes the angular motion of the energy carrier (tractor) around its own center of mass. The last fourth equation (7) determines the regularity of the angular motion of the harvester around its center of mass.

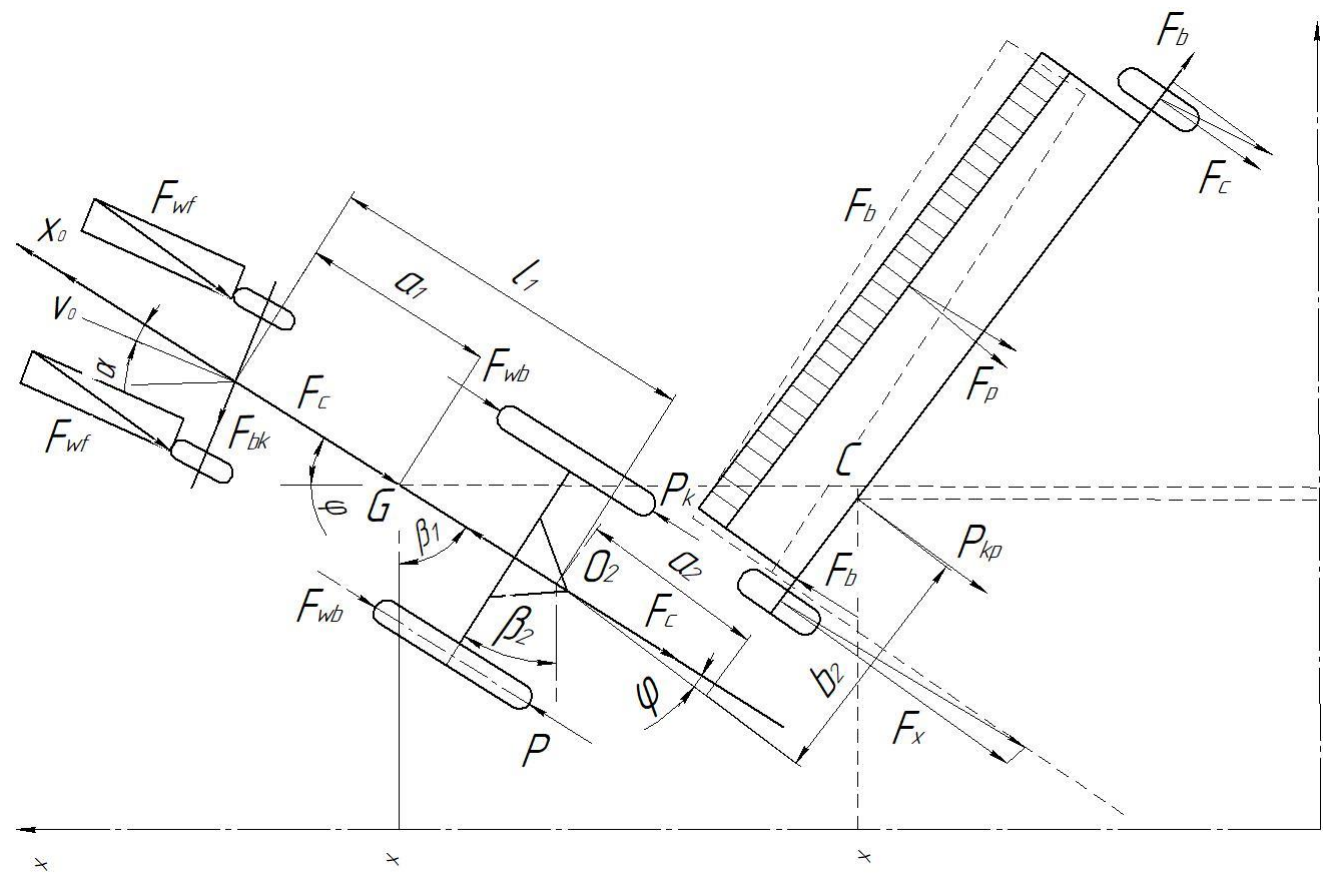

Fig. 3. Scheme of forces acting on the energy carrier (tractor) and harvester when mowing bread mass

An important step in improving the movement of the MTU harvester during field work is to improve the trajectory of its movements with a decrease in energy consumption of the process. If possible, in serial MTU harvesters with a side harvester hitch, the operator performs control actions to reduce the length of the perimeter of the MTU trajectory. Its effect on the front steered wheels is to reduce the disturbing forces that occur as accidental actions of force factors during field work. Thus, the operator is an important closing link in a complex dynamic socio-technical system "man-unit".

However, the MTU harvester can be considered as an object of control to create an additional system of automatic stability control. The analysis of the dynamics of the serial harvester MTU showed that the stability of its movement in the direction of the speed $V_{0}$ is due to the balance of resistance to 
the movement of the harvester and the lateral reactions of the forces acting on the front steered wheels. And the size of the latter, which balances the movement, depends on the position of the front wheels. That is, from the angle of their deviation $\alpha$ and is set directly by the operator - the tractor driver with the help of the steering wheel.

The disadvantages of such management are the need for active human intervention in the process of moving the MTU. Constant balancing of movement on the verge deviations of the unit in one direction or another, makes managing them quite hard work, and deviation from the ideal straight path brings additional economic costs. This method of controlling the stability of the serial unit generates undesirable forces of resistance to its movement to create the torque, which compensates for the moment of resistance to movement of the harvester. However, the sum of these points increases the overall resistance to the movement of the MTU. Thus, ensuring the straightforwardness of the MTU in the forward direction along the field requires the irrational use of additional energy resources.

Satisfactory controllability of the AIT harvester with a side hitch can be achieved by various technical means related to the improvement of both the power tool and the harvester itself. The solution should be considered ideal when the harvester moves across the field near the energy carrier (tractor) in parallel trajectories close to a straight line. At the same time energy consumption for performance of the technological process and moving of the unit becomes the minimum.

One of the effective ways to implement this idea can be to provide a controllable drive to the field wheel of the harvester. In this case, the wheel becomes driven with controlled torque applied to it. The scientific interest in solving this problem is related to the construction of a model of the control device, which must necessarily convert the input parameters of the perturbing effect of loads into the output of the MTU. The angle of deviation of the harvester $\varphi$ from the course of the direction of the power unit (tractor) can be used as the input parameter of the automated control system in relation to the harvesting unit. It is necessary that the increase of this angle according to a certain law changes the initial value of the control device, which leads to an increase in the torque on the field wheel of the harvester.

Activation of the drive on the field wheel of the harvester is directed to improvement of the management of MTU and to increase of the basic indicators of its work. However, the controlled drive requires the development of a special system of automatic control of its torque depending on the degree of deviation from the trajectory. Thus, there is a need to develop such a control system for the movement of the harvester with a side hitch on the power tool (tractor).

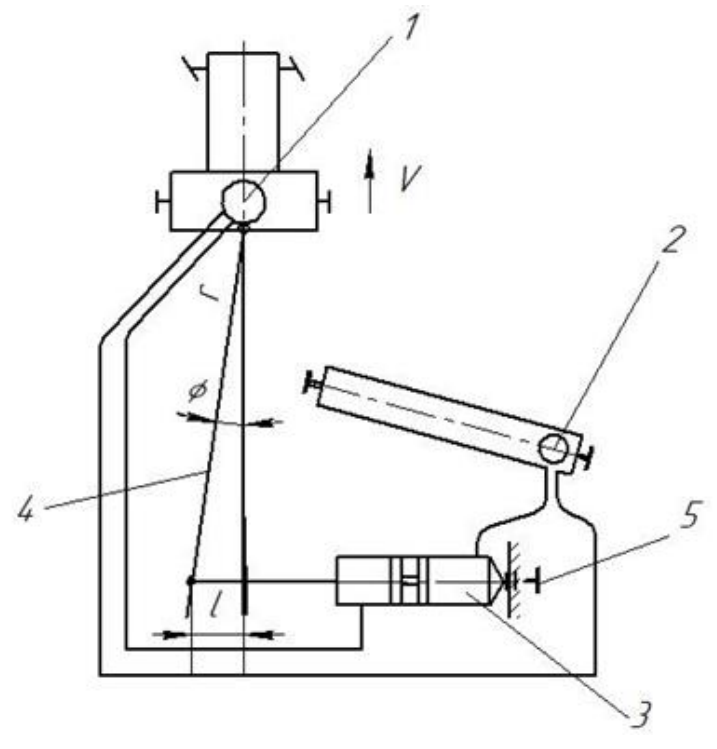

Fig 4. Scheme of the system of automatic control of movement of the harvester with a side hitch:

1 - hydraulic pump of a tractor; 2 - hydraulic motor of the drive wheel the harvester; 3 - liquid distributor (regulator) of the automatic system; 4 - mechanical system (meter);

5 - sensor of the initial level of deviation $l_{o}$

Such a system (Fig 4) should include controls, as well as an actuator in the form of a hydraulic motor. When creating an automatic system, it is necessary to establish its structure and identify the basic 
parameters by which the control process is organized. According to the presented scheme, the automatic system works as follows. As a result of the difference between the torque of the hydraulic motor and the moment of resistance to the movement of the harvester, there is a deviation from the course of the direction of the MTU. This deviation is transmitted through the mechanical lever system to the rod of the fluid distributor, which moves in its housing by a certain amount $l$. The mechanical system is a measuring body that converts the angular displacement of the harvester into a linear displacement of the distributor rod. In addition, the mechanical fluid distributor system has the ability to adjust to the desired initial level of the position of the rod $l_{0}$ relative to the housing. This achieves the setting of the specified level of deviation of the harvester.

\section{Results and discussion}

The stability of the movement was evaluated by the trajectory characteristics of the movements of the serial and experimental MTU. The greatest difficulty in driving MTU with a side hitch harvester is the formation of joints between adjacent passages. In the general case, areas of gaps and overlaps can be observed at the joints. Overlapping leads to a decrease in the productivity of the unit and to unnecessary fuel consumption, and omissions to crop losses. Since losses must be minimized, when driving the unit in the field, there is a need to form joints with guaranteed overlap. The analysis is based on the trajectory itself, which is represented by deviations from the baseline. Comparison of the deviations of the serial and experimental MTU obtained under identical conditions of the experiment showed a decrease in the deviation angle by an average of $1^{\circ} 10^{\prime}$ or more than $32 \%$. The obtained result indicates an improvement in the smoothness of the MTU with the introduction of an active controlled drive. Comparative general energy assessment of serial and experimental harvesting energy in real field operation conditions is carried out on fuel consumption and productivity.

Comparative energy evaluation of serial and experimental units is shown in Table 1.

Results of comparative energy assessment of work of harvesting MTU

Table 1

\begin{tabular}{|c|c|c|c|c|c|c|c|c|c|}
\hline \multirow{2}{*}{$\begin{array}{c}\text { MTU } \\
\text { speed, } \\
\mathbf{m} \cdot \mathbf{s}^{-1} \\
\left(\mathbf{k m} \cdot \mathbf{h}^{-1}\right)\end{array}$} & \multicolumn{2}{|c|}{$\begin{array}{c}\text { Average fuel } \\
\text { consumption per } \\
\text { experiment, } g\end{array}$} & \multirow{2}{*}{$\begin{array}{c}\text { Average } \\
\text { time of the } \\
\text { experiment } \\
t_{d}, \text { sec }\end{array}$} & \multicolumn{2}{|c|}{$\begin{array}{c}\text { MTU } \\
\text { performance, } \\
W, \mathbf{h a}^{-h^{-1}}\end{array}$} & \multicolumn{2}{|c|}{$\begin{array}{c}\text { Hourly fuel } \\
\text { consumption, } \\
G_{h}, \mathrm{~kg}^{\circ} \mathbf{h}^{-1} \text {. }\end{array}$} & \multicolumn{2}{|c|}{$\begin{array}{c}\text { Specific fuel } \\
\text { consumption, } \\
G_{p}, \mathrm{~kg} \cdot \mathrm{ha}^{-1}\end{array}$} \\
\hline & ser & exp & & ser & $\exp$ & ser & exp & ser & exp \\
\hline 0.3 & 2 & 24 & 2 & 0.72 & 0. & 3.25 & 3.2 & 4. & 4.31 \\
\hline $0.55(2.0)$ & 202 & 198 & 179 & 1.11 & 1.17 & 4.06 & 3.98 & 3.64 & 3.4 \\
\hline $1.3(4.7)$ & 12 & 121 & 76 & 2.61 & 2.75 & 5.97 & 5.73 & 2.28 & 2.1 \\
\hline $1.43(5.1)$ & 12 & 115 & 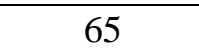 & 2.85 & 3,0 & 6.76 & 6.37 & 2.37 & 2.12 \\
\hline $2.1(7.6)$ & 128 & 107 & 48 & 4.23 & 4.46 & 7.6 & 6.42 & 1.8 & 1.44 \\
\hline
\end{tabular}

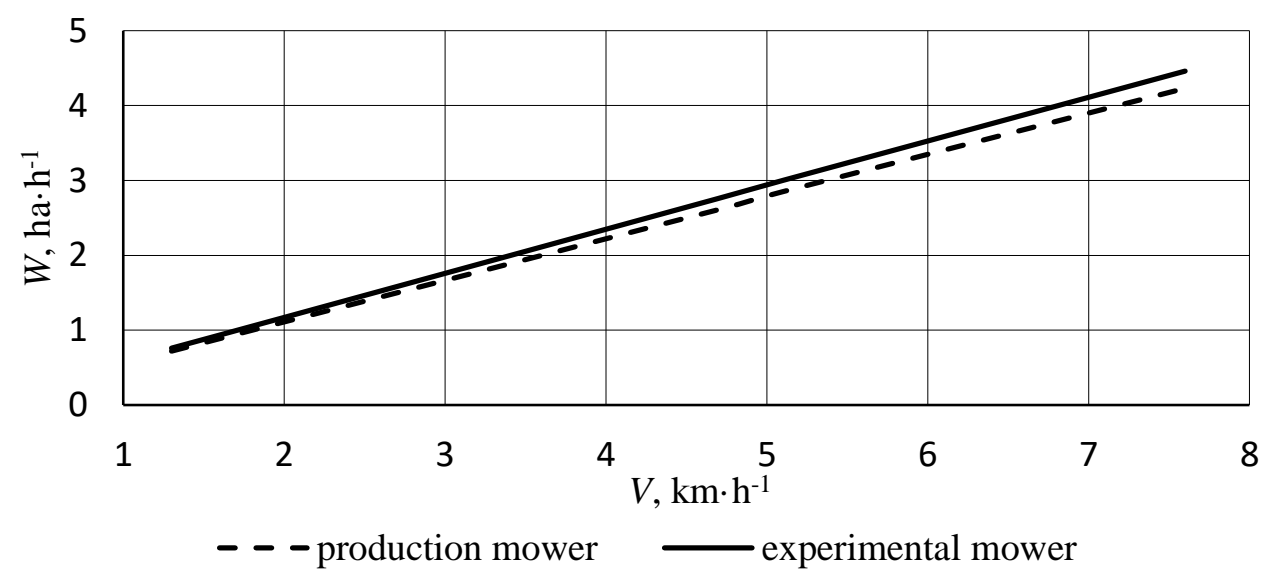

Fig 5. Comparative dependence of unit productivity on linear speed of movement of serial and experimental unit, respectively 
After analyzing the data in the table, the dependence of the unit performance on the speed change is established and shown in Fig. 5. The experimental unit showed the best indicators, which improve the performance of the unit by $5.4 \%$. In this case, according to the table, the overall reduction in the fuel consumption is $15 \%$. Statistical analysis allowed to determine significant regression coefficients, and to obtain simplified equations that adequately describe the process with a given $95 \%$ probability.

\section{Conclusions}

As a result of the research it was found that:

1. The automated system for ensuring the stability of the MTU harvester with a side hitch can be developed on the basis of the synthesis of mechanical and hydraulic subsystems with the activation of the drive on the field wheel.

2. The trajectories of both serial and experimental harvesting units are characterized by the presence of constant components of deviations (trends). According to Student's criteria, the introduction of an active drive on the field wheel reduces the value of the trend from $t=7.31$ to $t=5.36$.

3. The introduction of an active drive on the field wheel of the harvester stabilizes the movement of the AIT. The root mean square deviation of the trajectory decreases from $\sigma_{\text {ser }}=0.049 \mathrm{~m}$ to $\sigma_{\text {exp }}=0.040 \mathrm{~m}$, and the angle of deviation of the trajectory from the rectilinear decreases by an average of $1^{\circ} 10^{\prime}$.

4. It has been experimentally proven that with the introduction of a controlled drive on the field wheel of the harvester, the productivity increased by $17 \%$, and the specific fuel consumption decreased by $9 \%$.

\section{References}

[1] Bulgakov V., Pascuzzi S., Nadykto V., Ivanovs S. Mathematical Model of the Plane-Parallel Movement of an Asymmetric Machine-and-Tractor Aggregate. Agriculture, 2018. [online] [21.03.2021] Available at: https://www.mdpi.com/2077-0472/8/10/151

[2] Adamchuk V., Petrychenko I., Korenko M., Beloev H., Borisov B. Study plane-parallel motion movement combined seeding unit. Mechanization in agriculture \& Conserving of the resources, vol. 64 (2018), Issue 6, pg(s) 184-187.

[3] Antoshchenkov R. Results of mathematical model of plane-parallel motion multielement tractor unit March 2015 Actual directions of scientific researches of the XXI century theory and practice 3(2):15-19 March 2015 Actual directions of scientific researches of the XXI century theory and practice 3(2):15-19. [online] [21.03.2021] Available at:

https://www.researchgate.net/publication/313273832_Results_of_mathematical_model_of_plane

[4] Adamchuk V., Bulgakov V., Holovach I., Kuvachov V. Mathematical modelling of the movements of tractor working tools in controlled traffic farming. Agricultural Science and Practice, 2017, vol. 4, No. 2, pp. 64-73.

[5] Ivanovs S., Bulgakov V., Adamchuk V., Kyurchev V. Experimental research on the movement stability of a ploughing aggregate, composed according to the "push-pull" scheme. INMATECH Agricultural Engineering, 2018, vol. 56, No. 36, pp. 9-16.

[6] Troyanovskayaa I., Pozinb B., Noskovb N. Ploughing Tractor Lateral Withdrawal Model. International Conference on Industrial Engineering, ICIE 2017. Procedia Engineering 206 (2017). pp. 1540-1545. Available at: [online] [21.03.2021]

https://www.researchgate.net/publication/320942683_Plou

[7] Mohammadreza A., Gholamhossein S., Abbaspour G., Apolonio H. Measuring and Comparing Forces Acting on Moldboard Plow and Para-Plow withWing to Replace Moldboard Plow with ParaPlow for Tillage and Modeling It Using Adaptive Neuro-Fuzzy Interface System. Agriculture, 633. 2020. Available at: https://www.mdpi.com/2077-0472/10/12/633/pdf

Chupin P., Golovin A., Soyunov A. Determination of balanced plow movement for diamond plowing. ESCGAP 2020 IOP Conf. Series: Earth and Environmental Science, 659. 2021. [online] [21.03.2021] Available at: https://iopscience.iop.org/article/10.1

[8] Tajanowskij G., Wojciech T., Mariusz S. Modeling of kinematics of movement on turn of a wheeled tractor with a hinge-operated drawbar on semitrailer TEKA. Commission of motorization and energetics in agriculture, 2012, vol. 12, No. 1, pp. 293-300. 